\title{
Reality TV - Definition und Merkmale einer erfolgreichen Genrefamilie am Beispiel von Reality Soap und Docu Soap
}

\author{
Elisabeth Klaus / Stephanie Lücke
}

Traumquoten für "Deutschland sucht den Superstar" (RTL), der Grimme-Preis für „Schwarzwaldhaus 1902" (SWR), Start der vierten Staffel von „Big Brother" (RTL II): Alltagspersonen und Alltagsthemen haben im Fernsehen Hochkonjunktur. Mit Bezug auf frübere Studien definiert der Beitrag Reality TV als eine höchst lebendige Genrefamilie, die zablreiche Genres in sich vereint, darunter die „Gerichts-Show“, „Daily Talks“, „Personal Help-Shows" und die aktuellen "Casting-Shows". Am Beispiel der Hybridgenres Reality Soap und Docu Soap, die das deutsche Reality TV im Übergang zum 21. Jahrbundert besonders beeinflussten, werden die wichtigsten Merkmale der Genrefamilie aufgezeigt. Diese lassen sich inhaltich durch bewusste Grenzübertretungen charakterisieren, wie die Vermischung von fiktionalen und nicht-fiktionalen Elementen, von Authentizität und Inszenierung, Alltag und Exotik sowie Information und Unterhaltung. Formal sind sie durch gleiche Inszenierungsstrategien geprägt, und zwar Personalisierung, Emotionalisierung, Intimisierung, Stereotypisierung und Dramatisierung. Der Beitrag versteht sich als Zwischenbilanz einer in der deutschen Fernseblandschaft nicht mehr weg zu denkenden Genrefamilie mit ungebrochenem Entwicklungspotenzial.

Keywords: Casting-Show, Daily Talk, Docu Soap, Genres, Gerichts-Show, Inszenierungsstrategien, Personal Help-Show, Reality Show, Reality Soap

\section{Einleitung}

RTL hat es wieder einmal geschafft, eine Fernsehsendung zum Thema einer ganzen Nation zu machen. Die Casting-Show „Deutschland sucht den Superstar“ (DSDS), die von November 2002 bis März 2003 ausgestrahlt wurde, lockte bis zu 13 Millionen Zuschauerinnen und Zuschauer bei zeitweise über 40\% Marktanteil in der werberelevanten Zielgruppe der 14- bis 49-Jährigen vor den Fernseher (vgl. etwa Graff 2003, 10; Hoff 2003, 19). Die BILD-Zeitung füllte ihre Schlagzeilen mit Berichten über die angehenden „Superstars“ Daniel, Juliette, Alexander und Co.. Die Casting-Show ist nicht die erste ihrer Art - in den vergangenen Jahren brachten auch „Popstars“, „Popstars 2“ oder „Teenstar“ (alle RTL II) gute Quoten und erfolgreiche Bands wie die „No Angels“ oder „Bro’Sis“ hervor. ${ }^{1}$ Jedoch erreichte keine annähernd so viel Präsenz in den Medien und Köpfen der Menschen wie „Deutschland sucht den Superstar“. Die Sendung wurde wie „Big Brother“ (RTL II/RTL), dessen vierte Staffel im März 2003 startete, zu einem nationalen Medienereignis.

Wir argumentieren im Folgenden, dass beide Formate das Ergebnis eines Ausdifferenzierungsprozesses des Reality TV sind. Reality TV hat seit den 90er Jahren wesent-

1 Ein anderer Versuch von RTL, „Deine Band“, scheiterte an zu geringer Zuschauerresonanz die Reality Soap wurde nach acht Folgen abgesetzt, geplant waren zehn Folgen (vgl. Lücke 2002, 93f.). 
liche Entwicklungen im deutschen Fernsehen sowohl aufgegriffen als auch selbst vorangetrieben, wie wir am Beispiel zweier Genres, der Docu Soap und der Reality Soap, herausarbeiten. Die Collage aus nicht-fiktionalen und fiktionalen Bestandteilen, die Hinwendung zu alltäglicheren, der Lebenswelt der Zuschauerinnen und Zuschauer entnommenen Themen und damit einhergehend die emotionalisierte Darstellung des Privaten und Intimen in der Öffentlichkeit gehören zu den wesentlichen Merkmalen des Reality TV. Deren immer neue Mischung und Kopplung mit anderen Genres besitzt offensichtlich ein ungebrochenes Innovationspotenzial, wie der Erfolg der Casting-Shows oder auch der Gerichts-Shows zeigt.

Im Weiteren stellen wir verschiedene Definitionsversuche zum Reality TV vor und entwickeln auf der Basis ihrer kritischen Lektüre eine aktualisierte, den heutigen Ausprägungen der Genrefamilie entsprechende Definition. Im Anschluss greifen wir Docu Soap und Reality Soap als wichtige Genres des Reality TV heraus und analysieren an diesen Beispielen, welche gemeinsamen Merkmale das Reality TV ausweist.

In diesem Beitrag geht es somit wesentlich um Definitionsmerkmale von Genres. Dieser Begriff wird sehr häufig verwendet, manchmal synonym, manchmal in Abgrenzung zu ähnlichen Begriffen wie „Gattung“ oder „Hybridgenre“. Wir verstehen im Folgenden unter der Bezeichnung „Gattung“ in Anlehnung an Gehrau Begriffe, die „Fernsehangebote nach ihrer Form systematisieren und bezeichnen“, während „Genre“ „am Inhalt orientierte Untergruppen" der Gattungen sind (2001, 18; Hervorh. d. Verf.). Mit dem Begriff „Reality TV“ bezeichnen wir eine im deutschen Fernsehen verstärkt seit Beginn der 90er Jahre verbreitete Fernsehgattung, die in ihrer Form Elemente mehrerer anderer Gattungen, wie der Serie und der Dokumentation, aufweist. Insofern folgt Reality TV dem Trend der „Hybridisierung“ vieler Fernsehangebote, d. h. dem Phänomen, dass durch die Verknüpfung verschiedener Gattungs- oder Genrecharakteristiken neue Formate geschaffen werden (vgl. beispielsweise Turner 2001b, 6; Weischenberg 2001, 67; Neale 2001, 4; oder Bleicher 1999, 132). Die Gattung Reality TV hat sich, wie wir im Folgenden zeigen möchten, in eine Vielzahl unterschiedlicher Genres ausdifferenziert, so dass wir sie als eine „Genrefamilie“ bezeichnen möchten. Mit dem Begriff „Hybridgenre“ werden schließlich Genres bezeichnet, die unterschiedliche Gattungen und Genres zu einem neuen Format verknüpfen; diese Bedingung trifft auf die Genres des Reality TV zu. Angemerkt sei abschließend, dass die Zuordnung von Fernsehsendungen zu Genres im Idealfall das Ergebnis eines dynamischen Aushandlungsprozesses zwischen der Produktions- und der Rezeptionsseite ist und dass Rezipierende keineswegs so klar zwischen Form und Inhalt, zwischen Gattung und Genre differenzieren, wie dies in diesem Beitrag zum besseren Verständnis geschieht. ${ }^{2}$ Die Sicht der Rezipierenden findet jedoch im folgenden Beitrag keine Berücksichtigung.

2 Die Zuordnung neuer Fernsehprodukte zu einem Genre verbindet vor allem das Bestreben der Produzentenseite, Erwartungshaltungen der Zuschauer aufzubauen, und die strukturierende und orientierende Funktion für das Fernsehpublikum. Zur Bedeutung von Genres für Produzenten und die Publika beispielsweise Neale 2001, 3; Turner 2001a, 5 bzw. Fiske 1997, 109. Gattungen und Genres müssen im stets im Umbruch befindlichen Fernsehen als dynamisch und veränderlich gelten. 


\section{Ausdifferenzierung des Reality TV}

Reality TV wurde vor allem Anfang der 90er Jahre zum Gegenstand wissenschaftlicher Diskussionen, nachdem Varianten der in den USA entstandenen Gattung auch im deutschen Fernsehen zu sehen waren. Obwohl Sendungen wie „Polizeireport Deutschland“ (Tele 5), „K - Verbrechen im Fadenkreuz“ (SAT.1) oder „Kripo live“ (MDR) dem Prinzip des schon lange etablierten „Aktenzeichen XY - ungelöst“ (ZDF) folgten, lösten sie eine medienethische Debatte aus, in deren Folge sich mehrere Studien mit dem neuen Format beschäftigten. Claudia Wegener leistet 1994 in einer der ersten Arbeiten zum Reality TV eine Gattungseingrenzung, gestützt auf Interviews mit Redakteuren und Moderatoren von Reality TV-Sendungen. ${ }^{3}$ Als Charakteristika der Sendungen nennt Wegener die Darstellung von Emotionen, Personalisierung, Dramatisierung bzw. die Darstellung von Gewalt sowie Stereotypisierung (vgl. ebd., 43ff. bzw. 51ff.). Die gemeinsame Basis des diffusen „Wirklichkeitsfernsehens“ sei, dass tatsächliche Ereignisse - meist Katastrophen, Unfälle oder Verbrechen - nachgestellt oder durch Videoaufnahmen dokumentiert würden (vgl. ebd., 15f.). Für Wegener gehört demnach die Thematisierung und Darstellung von Gewalt zwingend zum Format Reality TV hinzu.

Gary Bente und Bettina Fromm schließen sich drei Jahre später der Definition von Wegener an. Sie untersuchen die Varianten des „Affektfernsehens“, eines neuen Genres, welches sie sowohl im Informations- als auch im Unterhaltungsbereich wiederfinden (vgl. 1997, 14). ${ }^{4}$ Als gemeinsame Merkmale von Sendungen des Affektfernsehens nennen sie Emotionalisierung, Personalisierung, Intimisierung und Authentizität. Zwar zählen sie zunächst das Reality TV anhand der erwähnten Merkmale zum Affektfernsehen hinzu, schließen es jedoch aus ihrer empirischen Untersuchung aus mit der Begründung, das von Wegener genannte Charakteristikum (Darstellung von Gewalt) sei beim Reality TV zentral, treffe aber auf die anderen Sendungen des Affektfernsehens nicht zu. Die Sendungen des Reality TV seien „eher randständig im Sinne der Definition, weisen jedoch einige Gemeinsamkeiten mit dem Affektfernsehens auf“ (ebd., 21). Schon damals war diese Argumentation jedoch wenig überzeugend, weil mit derselben Begründung andere Varianten des Affektfernsehens ausgeschlossen werden müssten, etwa Infotainment als einziges Genre, das im Beitrags-Stil präsentiert wird, oder Spielshows, weil sie im Gegensatz zu den anderen Formaten aus Game Show-Elementen bestehen. In einer späteren Studie subsummiert Fromm (1999) die verschiedenen Varianten des Affektfernsehens unter dem Begriff „intime Formate“. Reality TV bezeichnet sie nicht länger als „randständig“, sondern als „Vorläufer“ (ebd., 19) intimer Formate. Mit dieser Beurteilung wird jedoch die Bedeutung des Reality TV im Fernsehprogramm der späten 90 er Jahre unterschätzt. ${ }^{5}$

Die Forschungsgruppe um Peter Winterhoff-Spurk (1994) geht demgegenüber in ihrer von der Landesanstalt für das Rundfunkwesen Saarland (LAR) in Auftrag gegebe-

3 Sie zählt folgende Sendungen dazu: „Aktenzeichen XY... ungelöst“ (ZDF), „Polizeireport Deutschland“ (Tele 5), „Notruf“, „Auf Leben und Tod“, „Augenzeugen-Video“ (alle RTL), „Retter“, „K - Verbrechen im Fadenkreuz“ und „SK-15“ (alle SAT.1).

4 Zum Affektfernsehen zählen sie Affekt-Talks, Beziehungs-Shows, Spielshows, Suchsendungen, Konfro-Talks, Infotainment und Reality TV (vgl. ebd., 21).

5 Dass Reality TV im Fernsehen der 90er Jahre bedeutsam ist, zeigt die starke Ausdifferenzierung der Genrefamilie, vgl. Abbildung 1. 
nen Studie davon aus, dass es sich beim Reality TV um ein neues Fernsehgenre ${ }^{6}$ mit Unterkategorien handele (vgl. ebd., 205). Diese Unterkategorien teilen sie ein in kriminelles Verhalten, Unglücksfälle und nicht-kriminelles deviantes Verbalten. Die damals neuen „Suchformate“ „Vermißt“ (WDR), „Spurlos“ (RTL) und „Bitte melde Dich“ (SAT.1) sowie die Versöhnungsshows „Nur die Liebe zählt“ und „Verzeih mir“ (beide RTL) betrachten Winterhoff-Spurk und sein Team somit als Unterkategorie des Reality TV. Das von Wegener genannte Kriterium „Gewalt“ ersetzen sie durch „negative Deviationen des Alltäglichen“. Sendungen, in denen „Medienereignisse (Eheschließungen, überraschende Begegnungen mit Prominenten etc. vor laufender Kamera) inszeniert und ,live“ gezeigt werden“, wie etwa „Traumhochzeit“ oder „Verstehen Sie Spaß?“ (ebd.), zählen sie jedoch nicht zum Reality TV: Diese Ereignisse seien bereits in der Vergangenheit geschehen und täuschten lediglich eine Art „Live-Charakter“ vor (vgl. ebd., 206). ${ }^{7}$ Auch dieses Ausschlusskriterium kann jedoch nicht überzeugen, da die Ereignisse aller Sendungen des Reality TV in der Vergangenheit geschehen sind, auch wenn sie möglichst ereignis- und realitätsnah, teilweise auch mit Originalbildern, inszeniert werden. Eine Sendung wie „Verzeih mir“ unterscheidet sich in Bezug auf ihren Live-Charakter nicht von der „Traumhochzeit“ (RTL) oder dem „Flitterabend“ (ARD).

Für Angela Keppler gehören folgerichtig die genannten Sendungen zum „Realitätsfernsehen“ hinzu. Keppler hat 1994 eine erweiterte Definition des Reality TV vorgelegt, indem sie zwischen dem "narrativen Realitätsfernseben " und dem "performativen Realitätsfernseben " unterscheidet. In der deutschen Fernsehunterhaltung würden seit Anfang der 90er Jahre die Zuschauenden in neuer Weise zu AkteurInnen, und zwar als „Akteure ihres eigenen Lebens“ (1994, 7). Alltag und Alltagspersonen stünden sowohl bei den narrativen Formen, die tatsächliche Katastrophen reinszenierten, wie auch bei den glamouröseren Sendungen, die auf herausgehobene Aktionen setzten, im Mittelpunkt (ebd., S. 8f.). Im Unterschied zu Game- und Quizshows, die auch (z. B. mit einem Geldgewinn) in die Alltagswirklichkeit der Menschen eingreifen, werden bei Sendungen des performativen Realitätsfernsehens

„soziale Handlungen ausgeführt, die als solche bereits das alltägliche soziale Leben der Akteure verändern. Es sind die inzwischen schon kaum mehr zu überschauenden ,Kennenlern-' und ,Liebessendungen', die zur Gattung des performativen ,Reality-TV‘ gehören und die bei weitem populärste Form des Wirklichkeitsfernsehens darstellen." (ebd., 9. Hervorh. d. A.)

Auch Sendungen wie „Traumhochzeit“ oder „Verstehen Sie Spaß?“ sind demnach für Keppler Reality TV. Als Vorgänger des performativen Realitätsfernsehens sieht die Autorin die Daily Talks wie „Hans Meiser“, weil auch hier „echte Menschen in (...) echten Bedrängnissen zu Wort kommen“ (ebd., 44), die mit ihren Lebenserfahrungen und Alltagssorgen die Öffentlichkeit des Fernsehens suchten.

Insgesamt gelingt es Keppler, Reality TV einsichtig zu definieren und eine breite Grundlage für die Analyse der heutigen Ausprägungen und konstitutiven Merkmale dieser Genrefamilie zu legen. Reality TV hat demnach zwei Ausprägungen, das narrative und performative Realitätsfernsehen, die wir in Anlehnung an Keppler (ebd., 8f.) wie folgt definieren:

6 In Abweichung zum vorliegenden Beitrag bezeichnen sie Reality TV als „Genre“.

7 Die Autoren zählen diese Sendungen zu dem nicht näher eingegrenzten Genre „TV-Show“. 
Narratives Reality TV umfasst jene Sendungen, die ihre ZuschauerInnen mit der authentischen oder nachgestellten Wiedergabe realer oder realitätsnaher außergewöhnlicher Ereignisse nicht-prominenter Darsteller unterhalten.

Performatives Reality TV umfasst jene Sendungen, die eine Bühne für nicht-alltägliche Inszenierungen sind, jedoch zugleich direkt in die Alltagswirklichkeit nichtprominenter Menschen eingreifen.

Das narrative Reality $T V$ wird heute geprägt durch vier Genres: die gewaltzentrierten Sendungen, im Moment beispielsweise repräsentiert durch „Notruf“ (RTL) und „Kripo live" (MDR), die Real Life Comedy Formate, welche beispielsweise die US-Serie "Jackass“ (MTV) ${ }^{8}$ oder verschiedene Variationen des „Die dümmsten Verbrecher/ Tiere/ Unfälle der Welt“ (RTL II) umfassen, und die Gerichts-Shows, welche zu Lasten der Daily Talks im Nachmittagsprogramm seit 2001 an Sendezeit gewannen. ${ }^{9}$ Schließlich tragen in den Personal Help-Show ${ }^{10}$ Laien-Schauspieler zwischenmenschliche Konflikte aus und erhalten Rat und Hilfe von moderierenden Psychologinnen („Zwei bei Kallwass“, SAT.1), Medizinerinnen („Dr. Verena Breitenbach“, Pro7) oder Sozialpädagoginnen („Die Jugendberaterin“, Pro7), die eine Orientierungsfunktion als moralische Instanz erfüllen. Alle vier Genres drehen sich um außergewöhnliche Ereignisse des Alltags, wie Missgeschicke, Rechtsstreitigkeiten, Familien- oder Beziehungskonflikte sowie Verbrechen oder Unfälle, und gehören damit zum narrativen Reality TV.

Zum performativen Reality TV werden in der vorliegenden Literatur vier Genres gezählt. Es handelt sich um die Beziebungs-Shows („Verzeih mir“, „Nur die Liebe zählt“ - RTL), Beziebungs-Game Shows („Traumhochzeit“, RTL; „Herzblatt“, ARD), Daily Talks („Die Oliver Geissen-Show“, RTL; „Fliege“, ARD u. a.) sowie die Problemlösesendungen („Bitte melde Dich“, SAT.1; „Mit mir nicht!“, ZDF; „Wie bitte!?“, RTL).11 Unseres Erachtens erfüllen auch die relativ neuen Casting-Shows alle zur Definition des performativen Reality TV gehörenden Anforderungen. Beispielhaft wenden wir uns nun zwei Sendeformen genauer zu, die Ende der 90er Jahre entstanden sind und das Reality TV um ganz neue Aspekte erweiterten, dabei aber ganz unterschiedliche Resonanzen erzielten: die Docu Soap und die Reality Soap. Zuvor wollen wir aber in einem die bisherige Diskussion resümierenden Schaubild das Reality TV in seinen heutigen Formen vorstellen (siehe Abb. 1).

8 MTV stellte die Serie „Jackass“ nach 24 Episoden ein, nachdem konservative Politiker drohten, sie zu verbieten. Zuschauer hatten sich beim Nachahmen der Stunts schwer verletzt. Als „krönender Abschluss" kam am 27. Februar 2003 der Film „Jackass - The Movie“ in die deutschen Kinos, vgl. http://www.jackass.tv/main/index.php [Stand: 17. März 2003].

9 Es handelt sich um „Streit um Drei“ (ZDF), „Das Strafgericht“, „Das Familiengericht“, „Das Jugendgericht“ (alle RTL) sowie „Richterin Barbara Salesch“ und „Richter Alexander Hold“ (beide SAT.1). [Stand: 17. März 2003]

10 So bezeichnet Pro7 seine beiden zu diesem Format zählenden Sendungen, vgl. www.prosieben.de/talk/breitenbach/ [Stand: 17. März 2003].

$11 \mathrm{Zu}$ ihnen wird augenblicklich kein Format ausgestrahlt. Die Problemlösesendungen können als Vorläufer der Personal Help-Shows angesehen werden, da in beiden Fällen Moderatoren quasi anwaltschaftlich Probleme der Gäste aufgreifen und zu lösen versuchen. In den Personal HelpShows ist jedoch die narrative Struktur vorherrschend - die Fälle sind erfunden und werden nach Drehbuch von Laien-Schauspielern dargestellt. Es wird aus diesem Grund zum narrativen Realitätsfernsehen gezählt. 
Abbildung 1: Genre des Reality TV beute ${ }^{12}$
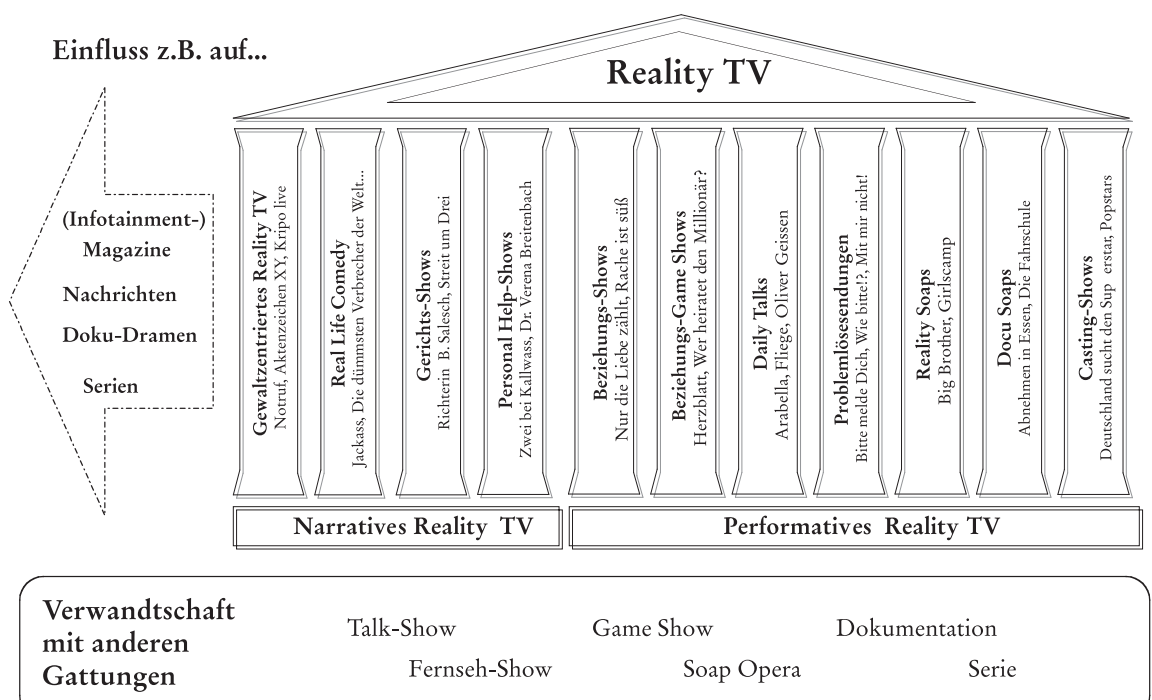

Talk-Show

Fernseh-Show
Game Show

Soap Opera
Dokumentation

Serie

Abbildung 1 zufolge zählen derzeit elf Genres zum Reality TV, wovon vier dem narrativen und sieben dem performativen Realitätsfernsehen zugeordnet werden können. Dabei schließt die Zuordnung eines Genres zum Reality TV die Zugehörigkeit zu anderen Genres nicht aus, alle Genres sind in hohem Maße hybridisiert: So gehören die Daily Talks zweifelsfrei auch zum Genre Talk Show. Real Life Comedy zeigt schon im Namen, dass es mit Comedy-Shows verwandt ist. Die Reality Soap und Docu Soap schließlich weisen Verwandtschaft zur Dokumentation, zur Soap Opera und im Fall der Reality Soap zu den Game Shows und Talk Shows auf. So wie andere Genres seine Entwicklung beeinflussten, ist das Reality TV nicht ohne Auswirkung auf andere Fernsehgenres geblieben. In Abbildung 1 werden die Sendeformen (Infotainment)-Magazine, Nachrichten und die Doku-Dramen erwähnt. Ein weiterer aktueller Trend zieht sich durch das Abendprogramm: Neu produzierte Serien setzen auf möglichst realistisch wirkende Geschichten (z. B. „Die Anstalt - zurück ins Leben“, SAT.1, seit 2002). Diesem Trend stehen Doku-Dramen gegenüber, die neben Zeitzeugenberichten und historischem Filmmaterial fiktionale Szenen integrieren (z. B. die Dokumentationen von Guido Knopp, ZDF, oder das zweiteilige Doku-Drama „Deutschlandspiel“, ARD).

12 Quelle: Lücke 2002, 51 (leicht modifiziert). 


\section{Der Aufstieg der Hybridgenres Docu Soap und Reality Soap}

Der zentrale Trend der Hybridisierung des Fernsehens der 90er Jahre zeigt sich nicht nur beim „Infotainment“, für das diese Eigenschaft namensgebend war, sondern auch bei den Genres des Reality TV - unter anderem deshalb, weil sich darin das Gewöhnliche und das Außergewöhnliche, Alltag und Inszenierung mischen und die geläufige Trennung zwischen nicht-fiktionalen und fiktionalen Produktionen, zwischen Unterhaltungs- und Informationssendungen bewusst durchbrochen wird. Reality TV ist zum Sammelbecken für neue, publikumswirksame Sendekonzepte geworden. Das ist vor allem im Übergang vom 20. zum 21. Jh. mit der fast zeitgleichen Etablierung zweier Genres deutlich geworden, die für die Ausdifferenzierung des Reality TV und seiner Abwendung von Gewalt und Katastrophen besonders einflussreich waren: Die Reality Soap, deren Prototyp „Big Brother“ (RTL II/RTL) viele Gemüter ähnlich stark erregte wie die Einführung der gewaltzentrierten Sendungen „Notruf“ (RTL), „Polizeireport Deutschland“ (Tele 5) und „Auf Leben und Tod“ (RTL) ein knappes Jahrzehnt zuvor, und die Docu Soap, die zu erbitterten Diskussionen unter Dokumentarfilmern über die Legitimation von Inszenierungen führte (vgl. Lücke 2002, 105f.).

Docu Soaps haben sich in Deutschland seit 1998 etabliert. ${ }^{13}$ Sie vermischen eine fiktionale Gattung (Serie) mit einer non-fiktionalen (Dokumentation). Inhaltlich zeichnen sich Docu Soaps dadurch aus, dass sich „normale Menschen“, also keine professionellen Schauspieler und Schauspielerinnen, freiwillig in ihrer gewohnten privaten oder beruflichen Umgebung von Kameras begleiten und filmen lassen. Die Akteure stellen ihren Alltag in der Fernseh-Öffentlichkeit dar und zeigen sich in privaten, nicht selten intimen Situationen. Die Docu Soap „Abnehmen in Essen“, bei der fünf übergewichtige Frauen ein Jahr lang bei ihren Versuchen begleitet wurden, dauerhaft Gewicht zu verlieren, wurde einer breiteren Öffentlichkeit bekannt, da sie im Jahr 2000 mit dem Grimme-Preis ausgezeichnet wurde. ${ }^{14}$

Reality Soaps gibt es im deutschen Fernsehen seit dem Start von „Big Brother“, dem Fernsehereignis des Jahres 2000.15 Ebenso wie bei Docu Soaps mischen ihre Regisseure Serie und Dokumentation zu einem neuen Produkt, darüber hinaus können die Reality Soaps jedoch Elemente der Talk Show und der Game Show enthalten. Anders als bei der Docu Soap ist ein zentrales Merkmal der Reality Soap, dass sich ihre ProtagonistInnen für die Zeit der Dreharbeiten in ein künstlich arrangiertes soziales Setting begeben, das immer zugleich eine Konkurrenzsituation beinhaltet, d. h. sie werden aus ihrem natürlichen Alltag in eine eigens für die Reality Soap entstandene Umgebung versetzt. ${ }^{16}$ Die Gestaltung dieses künstlichen Lebensraumes ermöglicht den Machern

13 Zur wissenschaftlichen Auseinandersetzung mit Docu Soaps siehe beispielsweise Lücke 2002, Eggert 1999 oder Bleicher 1999.

14 Vgl. http://www.grimme-institut.de/scripts/preis/preis.html [Stand: 17. Mai 2003].

15 Einen wissenschaftlichen Überblick über Reality Soaps, vor allem ihren bekanntesten Vertreter „Big Brother", bieten beispielsweise Balke 2000, Böhme-Dürr/ Sudhold 2001, Flicker 2001, Lücke 2002, Mikos et al. 2000, Schweer 2002 oder Weber 2000. Ein „Reality Lexikon“ mit „Echte Leute-TV von A bis Z" hat Feige 2001 zusammengestellt.

16 Seit dem Erfolg von „Big Brother“ waren Reality Soaps nur von Privatsendern produziert worden, die Landesmedienanstalten warnten die öffentlich-rechtlichen Sender, den Trend aufzugreifen. "Schwarzwaldhaus 1902“, eine SWR-Produktion für die ARD, durchbrach diese Schranke, indem der Sender eine gecastete Familie 10 Wochen lang auf einem Hof im Schwarzwald filmte - sie wurde auf den technischen Stand des Jahres 1902 zurück versetzt. Die viertei- 
der Reality Soap, extreme Lebensbedingungen zu schaffen und dadurch die Kommunikationen und Aktionen der Kandidatinnen und Kandidaten zu beeinflussen. Durch die Konkurrenzsituation steht deren Zusammenleben in der Spannung zwischen Gruppenharmonie und persönlicher Profilierung, so dass sich im Genre typische Elemente der Soap Opera, wie Melodramatik, Streit, Eifersucht und Neid, Versöhnung und Rivalität, finden. Auch wenn Docu Soap und Reality Soap vor allem jüngere Zielgruppen ansprechen, sind es keineswegs traditionslose Genres, wie ein kurzer Überblick zeigt.

\section{Zur Geschichte der Docu Soap}

Das wahrscheinlich erste Fernsehexperiment, in dem Menschen über einen längeren Zeitraum mit Kameras in ihrem Alltag beobachtet wurden, fand bereits 1972 in den USA statt. Dabei begleitete ein Kamerateam sieben Monate lang eine amerikanische Familie. Im Anschluss wurden 300 Stunden Filmmaterial zu einer Dokumentation zusammen geschnitten. Dass die Eheleute Loud sich während der Dreharbeiten trennten, führte zu kontroversen Diskussionen über die Auswirkung der Kamerapräsenz auf das Familienleben (vgl. Baudrillard 1978, 44ff.). Die erste Docu Soap im deutschen Fernsehen hieß „Die Fußbroichs“ (Untertitel: „Die einzig wahre Familienserie“, WDR). Im Mittelpunkt der Serie stand eine Kölner Arbeiterfamilie, die in einer Art „teilnehmender Beobachtung“ (Müller 1995, 91) bei besonderen Familienereignissen von einem Filmteam begleitet wurde. Zwischen 1990 und 2002 entstanden so in 17 Staffeln 99 Folgen der Docu Soap. ${ }^{17}$ Ihre Autorin und Regisseurin Ute Diehl erhielt dafür 1992 den AdolfGrimme-Preis in Bronze. ${ }^{18}$ Trotz des regional großen Erfolges der „Fußbroichs“ - im Kölner Raum erlangte die Familie Kultstatus (vgl. o.V. 1992b, 215) - folgten zunächst keine ähnlichen Produktionen.

Der Anstoß für einen wahren Docu Soap-Boom ging vielmehr von Großbritannien aus, wo seit Mitte der 90er Jahre teils mit sehr hohen Marktanteilen Docu Soaps „am Fließband“ produziert wurden (vgl. Eggert 1999, 10ff.). Mitte der 90er Jahre strahlte die BBC die dokumentarische Serie „Children's Hospital“ aus. ${ }^{19}$ Geschichten von kleinen Patienten in einem Londoner Krankenhaus wurden in mehreren Erzählsträngen miteinander verwoben. Ermuntert vom Erfolg dieser Serie, gab die BBC weitere dokumentarische Serien in Auftrag: 1996 entstanden „Vet's School“ (über eine Schule für Tierärzte) und „The House“ (Geschichten rund um die Mitarbeiter des Royal Opera House in London). „Driving School“, eine Serie über Menschen auf dem Weg zum Führerschein, verhalf den Docu Soaps schließlich in Großbritannien

lige „Dokumentation“ erreichte durchschnittlich mehr als 6 Millionen Zuschauer. Anstatt öffentlicher Debatten erhielten Regisseur und Kameramann den Grimme-Preis 2003 in der Sparte „Information \& Kultur“: „Selten gelang die Vermittlung von Alltagskultur auf derart perfektem und zudem unterhaltsamem Niveau.“. Vgl. http://www.swr.de/schwarzwaldhaus1902/ sowie http://www.grimme-institut.de/ [Stand: 17. März 2003].

17 Mittlerweile ist die Serie auf Wunsch der Familie Fußbroich eingestellt worden. Eine SerienChronologie findet sich unter http://www.epguides.de/fussbroi.txt [Stand: 17. März 2003.]

18 Vgl. o. V. 1992a, 8 sowie die Chronik der Preisträger des Adolf-Grimme-Instituts, http://www. grimme-institut.de/scripts/preis/preis.html [Stand: 17. März 2003].

19 Die folgenden Informationen über britische Docu Soaps stammen, soweit nicht anders gekennzeichnet, von Eggert 1999, 14 - 26. 
zum großen Durchbruch. Am 15. Juli 1997 erreichte „Driving School“ bei über 12 Millionen Zuschauern einen Marktanteil von 53\%. Allein 1998 strahlte das britische Fernsehen insgesamt 75 Docu Soaps aus. Viele davon wurden in andere Länder exportiert oder dienten als Vorbild für die Umsetzung eigener Produktionen auch in Deutschland: Das ZDF wandelte „Children's Hospital“ 1998 in „OP. Schicksale im Klinikum“ ab, RTL ließ sich von „The Cruise“ inspirieren und drehte 1999 „Das Clubschiff“, eine Docu Soap über Besatzung und Gäste des deutschen Kreuzfahrtschiffs „Aida“. SAT.1 nahm im gleichen Jahr „Driving School“ zum Anlass, „Die Fahrschule“ zu drehen, und RTL II kopierte dasselbe Format 2000 mit „You drive me crazy“. Bis Mitte 2001 entstanden sowohl bei den öffentlich-rechtlichen Anstalten ARTE, ZDF, WDR und anderen Dritten Programmen als auch bei den Privatsendern SAT.1, RTL und RTL II zu unterschiedlichsten Themenbereichen 46 weitere Docu Soaps (vgl. Lücke 2002, 79).

\section{Zur Geschichte der Reality Soap}

Auch die Geschichte der Reality Soap beginnt nicht erst mit „Big Brother“. 1994 setzte der Filmproduzent Markus Peichl in Berlin „Das wahre Leben“ (Premiere Deutschland) nach US-amerikanischem und britischem Vorbild um („The Real World“, MTV (USA)/ „The Living Soap“, BBC 2 (GB)). Sieben einander unbekannte junge Leute im Alter zwischen 21 und 26 Jahren zogen für drei Monate als Wohngemeinschaft in ein Berliner Loft. Kameras nahmen mindestens zehn Stunden täglich WG-Leben auf, wobei die Bewohner ein Mitspracherecht darüber hatten, was gefilmt werden durfte. Die Kandidatinnen und Kandidaten wurden nach Telegenität und nach Merkmalen wie Geschlecht, Beruf, sexueller Orientierung, Nationalität und Charakter ausgesucht, um für „ausreichendes Konfliktpotential“ zu sorgen (vgl. Strittmatter 1994, 95). Der Spiegel nannte die Serie, deren Ähnlichkeit zu „Big Brother“ unverkennbar ist, eine „neue, absurde Variante von Reality-TV“ (o. V. 1994, 225). Obwohl zunächst umstritten war, ob Docu Soap und Reality Soap Genres des Reality TV sind, gelten vor allem letztere heute geradezu als der Prototyp der Gattung (z. B. Mikos et al. 2000, Feige 2001, Hohlfeld 2000). Stefan Niggemeier (2003) setzt Reality Soap und Reality TV sogar in eins, wenn er die Vorbereitungen zu „Big Brother 4“ mit „Reality-TV kehrt zurück“ kommentiert. Nach einem „Overkill“ im Januar 2001, als innerhalb eines Monats gleichzeitig „Big Brother 3“ (RTL II, RTL), „To Club“ (RTL II), „Der Frisör“ (RTL), "House of Love“ (RTL) und "Girlscamp“ (SAT.1) starteten, verschwand die Reality Soap zeitweise vom Bildschirm. Weil sich in der Zwischenzeit andere Genres (Gerichts-Show, Personal Help-Show und Casting-Show) etablieren konnten, stürzte das Verschwinden der Reality Soap aus dem deutschen Fernsehprogramm zwischen 2001 und 2003 nicht zugleich auch das Reality TV in die Krise. Weiter legt auch der anhaltende Erfolg der Reality Soap im Ausland nahe, dass das Genre auch bei uns wieder verlorenes Terrain zurück erobern wird.

\section{Konstituierende Merkmale des Reality TV}

Docu Soaps und Reality Soaps stehen im besonderen Maße für die Ausdifferenzierung und Weiterentwicklung des Reality TV. Ihre Analyse erscheint deshalb geeignet, um die Charakteristika der Genrefamilie genauer herauszuarbeiten. Die folgende Beschreibung der wichtigsten Merkmale des Reality TV beruht wesentlich auf einer Bestandsaufnahme aller bis Mitte 2001 in Deutschland gesendeten Docu Soaps und Reality Soaps (vgl. 
Lücke 2002). ${ }^{20}$ Dabei konzentrieren wir uns in der Darstellung, gestützt auf die dazu vorliegende Literatur, auf diejenigen Ergebnisse, die für Genres des performativen ebenso wie des narrativen Reality TV konstitutiv sind. Diese Charakteristika lassen sich in vor allem den Inhalt betreffende Grenzübertretungen und vor allem die Form betreffende Inszenierungsstrategien unterteilen.

\subsection{Grenzübertretungen}

Grenzübertretungen markieren das Innovative der neuen Genres: Reality TV löst als Sammlung von Hybridgenres vermeintliche Gegensätze von Authentizität und Inszenierung, Information und Unterhaltung, Alltäglichem und Außergewöhnlichem auf und vermischt diese zu neuen Fernseh-Produkten. ${ }^{21}$ Grenzüberschreitend sind diese Merkmale in einem doppelten Sinn: Sie gelten für alle zum Reality TV zählenden Genres und sie verlassen gleichzeitig bis in die 90er Jahre hinein geltende Konventionen. ${ }^{22}$

Zwischen Authentizität und Inszenierung: Als authentisch wird etwas bezeichnet, wenn es „echt, glaubwürdig“ ist (Meyers Lexikonredaktion 1995, 264). Eine Fernsehsendung halten Bente und Fromm dann für authentisch, wenn unprominente Menschen „wahre Geschichten“ erzählen und/oder vor der Kamera darstellen (vgl. 1997, 20). Herrmann (2002, 130f.) beschreibt Authentizität in Talk Shows so: „Nichtprominente Bürger erzählen von ihrem Schicksal. (...) Die Geschichten sind also nicht erfunden, sondern wahr (...).“ Als Vertreter des performativen Reality TV stellen auch Docu Soaps und Reality Soaps Geschichten und Erlebnisse gewöhnlicher Menschen in den Mittelpunkt. Laut Selbstaussage der Fernsehsender handelt es sich vor allem bei der Docu Soap um ein authentisches Genre. Der Sender RTL II ${ }^{23}$ betont in seiner Pressemitteilung zu „Reeperbahn“ die Authentizität der Serie: „Keine Szene wird gestellt, wir begleiten unsere Darsteller mit der Kamera und lassen sie einfach erzählen (...): ,Dialoge schreiben wir nicht, die liefern uns unsere Darsteller.“" Noch direkter formuliert das ZDF in der Pressemappe zu „OP. Schicksale im Klinikum“ (1998): Es „entstanden Ge-

20 Im Rahmen einer kommunikationswissenschaftlichen Abschlussarbeit wurde zwischen April 1998 (erste Docu Soap im deutschen Fernsehen) und Mai 2001 das Fernsehprogramm mehrerer Fernsehzeitschriften durchgesehen und auf dieser Basis, kombiniert mit Selbstauskünften der Fernsehsender ARD, ZDF, ARTE, aller Dritten Programme, RTL, RTL II, SAT.1, Pro Sieben sowie VOX, alle bis zu diesem Zeitpunkt ausgestrahlten Docu Soaps und Reality Soaps erfasst. Von allen ermittelten Sendungen (46 Docu Soaps sowie 11 Reality Soaps) wurden Pressematerial sowie die Einschaltquoten bei der Erstausstrahlung angefordert sowie Zahl und Länge der Folgen, Ausstrahlungszeitraum, Hauptpersonen und -schauplatz sowie die Produktionsfirma recherchiert. Anhand der Durchsicht einer Auswahl von Docu Soaps und Reality Soaps wurde überprüft, inwieweit die aus der theoretischen Literatur hervorgehenden Gattungsmerkmale des Reality TV, von Daily Soaps und Dokumentationen sich in Docu Soaps und Reality Soaps wiederfinden.

21 Dass Reality TV ebenfalls Privates öffentlich macht, zeigt dieses Kapitel. Allerdings gehen die Autorinnen davon aus, dass Fernsehsendungen nicht erst mit dem Beginn der Ära Reality TV Privates thematisierten, sondern dies seit ihren ersten Tagen tun, wie z. B. Bleicher oder Herrmann herausarbeiten (vgl. Bleicher 2002, 208ff.; Herrmann 2002, 39 - 51). Es handelt sich deshalb in unserem Sinne nicht um eine formative Grenzübertretung.

22 Wie beispielsweise die Trennung des Fernsehprogramms in fiktionale und non-fiktionale Produkte.

23 RTL II nimmt für sich in Anspruch, die erste Docu Soap („Reeperbahn!“) im Privatfernsehen produziert zu haben. 
schichten von Menschen, deren Alltag geprägt ist vom Leben in der Klinik, meist über einen längeren Zeitraum. Nichts wurde dazu erfunden. Nichts nachgestellt, nichts inszeniert. Authentische Geschichten."

Solche Pressemitteilungen verbreiten jedoch nur eine Hälfte der Wahrheit. Auch in den Docu Soaps geht es - ebenso wie in den anderen Genres des performativen Reality TV, wie Herrmann $(2002,131$ f.) beispielhaft an den Daily Talks zeigt - nicht nur um die Darstellung realer Geschehnisse, sondern auch um ihre möglichst geschickte und spannende Inszenierung. So geben einige RegisseurInnen offen zu, dass sie Szenen nachstellen lassen oder in die Handlungen ihrer Protagonisten eingreifen, und zwar unabhängig davon, ob sie für einen öffentlich-rechtlichen oder privaten Sender arbeiten. Differenzierungen zeigen sich eher im Ausmaß der Eingriffe. So bekennt sich Bettina Böttinger, verantwortlich für zwei Staffeln „Ein Heim für alle Felle“ (WDR), in der Pressemappe zur Docu Soap dazu, nicht nur Szenen aus der Realität zu verarbeiten. Im Interview über die Dreharbeiten äußert sie sich: „Manchmal sind wir ihnen [den Mitarbeitern des Tierheims, Anmerkung d. A.] sicherlich auf die Nerven gegangen, wenn sehr bestimmt gefragt wurde, ob sie die eine oder andere Begebenheit mal eben nachspielen könnten und zwar ganz natürlich ...“ (2000, 7). Susanne Abel , Regisseurin von „Die Fahrschule“ und „Die Skischule“ (beide SAT.1), interessiert sich hingegen gar nicht erst für den „grauen Alltag“: „... wir zeigen Trudchen Müller nur, wenn es kracht. (...) Die reine Realität ist so spannend nicht, aber wenn man das flott montiert, kann das sehr spannend sein. (...) Wenn ich mit der Kamera komme, ist die Realität schon verändert." (zit. in Hoff 2000, 22).

Noch stärker kommt der Inszenierungscharakter in den Reality Soaps zum Ausdruck. Lothar Mikos hat vermerkt, dass es sich bei „Big Brother“ um „(...) ein um die Inszenierung von Authentizität bemühtes, auf die Alltagswelt von Zuschauern und Kandidaten Bezug nehmendes Format [handelt], das zum performativen Realitätsfernsehen gezählt werden kann." (Mikos et al. 2000, 28f.; Hervorh. d. A.). Mikos hat herausgearbeitet, dass „Big Brother“ und seine Nachfolger Authentizität nur vermitteln wollen, während im Hintergrund meist unsichtbar die Fernsehmaschinerie dafür sorgt, dass alles „richtig“ in Szene gesetzt wird. Die Montage einer zusammenhängenden Sendung aus der Fülle des zur Verfügung stehenden Materials erlaubt es ohne großen Aufwand, dem tatsächlichen Geschehen einen dramaturgischen roten Faden zu verleihen. Doch auch hier gibt es Hinweise, dass manchmal direkt nachgeholfen wird: Beispielsweise berichtete ein Teilnehmer der Reality Soap „Inselduell“, dass die Kameraleute häufig das Nachspielen von Szenen verlangten, deren Aufnahme schief gegangen oder verpasst worden war. Die Kandidatinnen und Kandidaten hätten sich vergeblich gegen diesen „Betrug“" gewehrt. ${ }^{24}$

Docu Soaps und Reality Soaps bewegen sich im Spannungsfeld zwischen Authentizität und Inszenierung, zwischen Realität und Fiktion. Sie möchten den Schein von Authentizität aufrecht erhalten, während sie tatsächlich Realität inszenieren. Heinrich Pachl, Regisseur der WDR-Docu Soap „Durchboxen“, nennt diesen Prozess in der Pressemappe „waschen, färben, föhnen, also arrangieren und stylen“ (o. J., 14). Die Wiedergabe vermeintlich authentischer Erlebnisse, die jedoch in eine feste Dramaturgie eingebunden sind, ist ein wesentliches Merkmal aller Genres des performativen wie narra-

24 Es handelt sich um den 51-jährigen Peter Pfaff, den ältesten Kandidaten des „Inselduells“, der von seinen Erfahrungen auf der Tagung „Das Fernsehen als Labor“ der Evangelischen Akademie in Hofgeismar (März 2001) berichtete. 
tiven Realitätsfernsehens. Für Daily Talks, Beziehungs-Shows oder das gewaltzentrierte Reality TV gilt gleichermaßen, dass die Geschichten aus dem echten Leben „normaler Menschen“ gegriffen sind und somit der Realität entstammen. Die Casting-Show „Deutschland sucht den Superstar“ zeigt, wie zunächst unbekannte Jugendliche versuchen, sich ihren Traum zu erfüllen und ein Superstar zu werden. Bei allen Formaten des Reality TV handelt es sich um eine verwirrende Mischung aus Realität und Inszenierung. Die „echten“ Richter der Gerichts-Shows verhandeln erfundene Fälle mit Laien-Schauspielern, die Ärztin erteilt ihre Ratschläge in vom Drehbuch vorgegebenen Konflikten, der Hergang von Unfällen wird fernsehgerecht nachgedreht, die schlagfertigen Antworten bei „Herzblatt“ von der Redaktion vorgegeben (vgl. Nolda 1996, 26). Neuberger $(1994,69)$ spricht von „Teil-Inszenierungen“ von Ereignissen: „Sie sollen den Anschein von Authentizität behalten und andererseits die Widerspenstigkeit der Realität durch Inszenierung berechenbar machen. "Dass die Realität in den Sendungen des Reality TV eben nicht nur abgebildet, sondern bearbeitet und verändert wird, macht aus ihren Inszenierungen realer Begebenheiten ein Stück Fiktion.

Zwischen Information und Unterhaltung: Bis in die 80er Jahre wurde in den öffentlich-rechtlichen Sendeanstalten strikt zwischen Informations- und Unterhaltungsproduktionen getrennt. Während anerkannt war, dass auch Informationssendungen unterhaltend aufbereitet sein mussten, ist der Nachweis, dass populäre Unterhaltungssendungen auch informieren, erst im Rahmen der neueren Publikumsforschung erbracht worden. In ihrer Zusammenfassung hat Elisabeth Klaus (1996) gezeigt, dass realitätsbezogene Genres, denen die Informationsfunktion zugeordnet wird, und fiktionale Genres, die mit Unterhaltungsfunktionen verbunden werden, sich in wesentlichen Teilen überschneiden und den Zuschauerinnen wie Zuschauern sowobl Information als auch Unterhaltung bieten. Wie das Infotainment zuvor, durchbrechen Docu Soap und Reality Soap bewusst den Dualismus von Unterhaltung und Information. Sie unterhalten durch die Darstellung alltäglicher Probleme von Menschen „wie du und ich“ und durch die Präsentation ihrer Missgeschicke oder Gefühlsausbrüche, ihrer komischen oder dramatischen Erlebnisse. Sie steigern die Spannung, indem schwierige Aufgaben gelöst oder menschliche Grenzsituationen bewältigt werden müssen. Damit informieren sie gleichzeitig nicht nur über Wissenswertes rund um Alltagsthemen - Schwangerschaft und Geburt, Partnervermittlung, Abnehmen - sondern vermitteln vor allem Wissen über den menschlichen Umgang miteinander: die Austragung eines Konflikts und Möglichkeiten der Versöhnung, des Trostes, die Bewältigung von Freude und Leid, die Legitimität von Liebe und Hass. Docu Soaps und Reality Soaps zeichnen sich nicht dadurch aus, dass sie Unterhaltendes mit Informationen anreichern oder Informatives unterhaltend aufbereiten, vielmehr lassen sie sich nur in der Grenzauflösung bestimmen.

Für alle Genres des performativen wie narrativen Reality TV gilt, dass sie die Grenze zwischen Unterhaltung und Information bewusst übertreten. Werner Früh et al. schlagen beispielsweise vor, im gewaltzentrierten Reality TV eine Sonderform des Infotainment zu sehen (vgl. 1996, 429). Als Kennzeichen der Information nennen sie „Service"-Teile, welche u. a. Informationen über Erste Hilfe-Maßnahmen oder die Arbeit der Feuerwehr integrieren. Die echten Richter und Anwälte der Gerichts-Shows sollen beiläufig Informationen über Fallstudien der deutschen Rechtsprechung vermitteln, und moderierende Psychologinnen, Medizinerinnen und Sozialpädagoginnen sorgen dafür, dass in den Personal Help-Shows die Auseinandersetzungen und die Verhandlung der Konflikte einen professionellen Anstrich bekommen und eine Orientierungsfunktion für die Rezipienten erfüllen können. Unumstritten, und von der Medienkritik besonders herausgestellt, ist demgegenüber die Unterhaltungsfunktion des Reality TV: Sie 
manifestiert sich durch den Einsatz dramaturgischer Mittel wie Emotionen (wie in Beziehungs-Shows, Daily Talks oder Personal Help-Shows), Komik (in Real Life Comedy) oder Dramatik (wie in gewaltzentriertem Reality TV oder Gerichts-Shows), die Nähe zur Fernseh-Show oder zur Serie.

Die heute geläufigere Doppelfunktion des Reality TV wurde aber nicht von Anfang an erkannt. Udo Michael Krüger ordnet in seiner Studie über Gewaltdarstellungen im Fernsehen 1992 das gewaltzentrierte Reality TV in den Bereich „Information“ ein, neben Nachrichtensendungen, politischen und nicht-politischen Informationssendungen. Diese Einordnung sei durch die GfK-Sendekodierung erforderlich, die Reality TV als eine Unterkategorie zum Informationsangebot behandele (vgl. 1992, 73). In der Programmanalyse 2001 werden dagegen ohne nähere Begründung Teile der Sendungsform „Reality/Dokuinszenierung“ - zu dieser Kategorie zählt auch das gewaltzentrierte Reality TV - in die Sparte Information, andere Teile in die Sparte Unterhaltung eingeordnet (vgl. Krüger/Zapf-Schramm 2002, 188f.). Auf längere Sicht erscheint zweifelhaft, ob an der Trennung zwischen Unterhaltung und Information in den Produktionsabteilungen der Sendeanstalten und den Kategoriensystemen der Medienforschung auf Dauer festgehalten werden kann. Das Reality TV mit all seinen Genres untergräbt diese Grenzsetzung jedenfalls stetig.

Alltag und Exotik: Der Alltagsbezug ist eine der zentralen Charakteristika des Realitätsfernsehens. Unterschiedlich aufbereitet steht das Gewöhnliche, Alltägliche auch in Docu Soaps und Reality Soaps im Vordergrund. Sie zeigen „normale“ Menschen bei der Bewältigung von Lebensabschnitten, die fast jeder Zuschauer aus seinem eigenen Leben kennt - seien es die vier pubertierenden Freundinnen („Die Schiller-Gang“, WDR), Nachwuchs erwartende Pärchen („Schnulleralarm“, RTL II), arbeitssuchende Kleinstadt-Bewohner („Artern - Stadt der Träume“, MDR) oder flirtwillige Singles („House of Love“, RTL). Anders als in den Docu Soaps verlassen die KandidatInnen in den Reality Soaps zwar ihre Alltagswelt, müssen aber, damit die Inszenierung gelingen kann, diese als ihren Bezugspunkt und als Quelle der Identifikation behalten. Für die Darstellenden sowohl der Docu Soap als auch der Reality Soap gilt aber zugleich, dass sie durch die Fernsehpräsenz für eine Zeit lang aus diesem Alltag herausgerissen werden. Der Blick der Kamera macht sie zu ExotInnen des Alltags, stellt sie als Einzelne aus, die von Millionen bestaunt werden können. Das außergewöhnliche Fernsehereignis transformiert die Alltagsmenschen zu Medienstars, wenn auch häufig nur für kurze Zeit, und stellt damit einen erheblichen Eingriff in ihre Alltagswelt dar, wie er für die Genres des performativen Reality TV konstituierend ist.

Diese Grenzübertretung von Alltäglichem und Ungewöhnlichem ist für die Genres des Reality TV konstitutiv. Ob bei „Oliver Geissen“, „Das Jugendgericht“, „Dr. Verena Breitenbach“ oder „Herzblatt“: Die ProtagonistInnen sind allesamt Nicht-Prominente. ${ }^{25}$ Dies ist eins der prägnanten Charakteristika für das Realitätsfernsehen. Die Tatsache, dass die Darsteller „ganz normale“ Menschen und keine Schauspieler oder Prominenten sind, bedeutet für die Zuschauenden eine Abweichung von den sonst üblichen „perfekten“ Fernsehfiguren. Weiter beziehen sich die Sendungen des Reality TV auch auf besondere Situationen des Alltags dieser Menschen: Schwangerschaft, Krankheit, Freundschaft - es geht um ungezählte Situationen der Lebensbewältigung. Wiederum gilt aber, dass das Fernsehen die Alltagsbegebenheiten in herausgehobene Ereignisse und

25 Bei Gerichts-Shows und Personal Help-Shows stellen Laien-Schauspieler die Szenen dar. 
die Alltagsmenschen in Fernsehstars überführt. Die ausverkauften Fan-Magazine bei „Deutschland sucht den Superstar“, die vielen Fanclubs für die KandidatInnen, die Absatzzahlen ihrer ersten CD „United“, die Traumquoten - all das spricht dafür, dass in der jeweils spezifischen Mischung von Gewöhnlichem und Außergewöhnlichem, von Alltag und Exotik der Schlüssel zum Erfolg der Genres des Reality TV liegt.

\subsection{Inszenierungsstrategien}

Neben den vor allem den Inhalt betreffenden Grenzübertretungen sorgten vor allem die die Form betreffenden Inszenierungsstrategien des Reality TV immer wieder für Konfliktstoff. Die einzelnen Genres setzen zielgerichtet jene dramaturgischen Mittel ein, die für das Fernsehen der 90er Jahre konstituierend geworden sind. Es sind die Stilmittel der Personalisierung, der Emotionalisierung, der Intimisierung, der Stereotypisierung und der Dramatisierung. ${ }^{26}$

Personalisierung: Docu Soaps und Reality Soaps sind dadurch gekennzeichnet, dass ihre ProtagonistInnen als Persönlichkeiten inszeniert werden und deshalb potenziell Identifikationsfiguren für ihr Publikum darstellen können. Menschen erzählen nicht nur von ihrem Schicksal ${ }^{27}$, sondern sie erleben und erleiden es direkt vor der Kamera, so dass die ZuschauerInnen sie ein Stück ihres Lebensweges begleiten können. ProtagonistInnen wie Sabrina Begic („Abnehmen in Essen“, ARTE/ WDR und „Big Diet“, RTL II) oder Manu, Zlatko und Jürgen („Big Brother 1“, RTL II/RTL) wurden so zu Vorbildern oder auch zu Hassfiguren. Eine solche Personalisierung ihrer nicht-prominenten Teilnehmenden ist ein wesentliches Element aller Reality TV-Genres. In fast allen Sendungen werden intime, private Details und Gefühle erörtert, in Interviews erzählen die Opfer, Retter oder Zeuginnen „ihre Geschichte“ persönlich und entsprechend gefühlsbetont.

Emotionalisierung: Mit der Personalisierung ist die Emotionalisierung eng verbunden. Auch sie gehört zu den wesentlichen Gestaltungsmerkmalen von Docu Soap und Reality Soap. Das Schicksal eines krebskranken Mädchens im Krankenhaus („OP. Schicksal im Klinikum“, ZDF) oder die Reaktionen der jungen Frau, deren Freund auf einem Video mit einer Konkurrentin flirtet („Versuchung im Paradies“, RTL) stellen hochemotionale, melodramatische Situationen dar. Als Mittel zur Spannungssteigerung und zur emotionalen Bindung werden Cliffhanger und schnelle Schnitte eingesetzt, so dass die Sendungen ihr Soap-Label insgesamt zu Recht tragen. Fast alle Reality TV-Genres präsentieren gewöhnliche Menschen in einer außergewöhnlichen Situation, die sie bewältigen - indem sie ihren Partner um Verzeihung bitten, ein lange vermisstes Familienmitglied wieder treffen oder einen Heiratsantrag machen. In der Regel zeigen die Betroffenen ohne Hemmungen eigene Emotionen. ${ }^{28}$ Der Weinkrampf von Kandidat Daniel Küblböck beim Ausscheiden seiner Freundin Gracia aus der Casting-Show

26 Diese wurden in den Arbeiten von Wegener 1994, Bente/ Fromm 1997 sowie Fromm 1999 genannt.

27 Von ihren Schicksalen reden sie in den Daily Talks, wie beispielsweise Herrmann (2002, 149ff.) herausarbeitet. Sie wies dabei den Begriff der „Personalisierung“ als negativ konnotiert zurück und ersetzte ihn durch „personenbezogene Darstellung“ (ebd., 150).

28 Bei den häufig in Daily Talks vorkommenden, teils sehr privaten und emotionalisierenden Themenbereichen Beziehung/ Sexualität, Familie und Freundschaft sind oft Tränen oder Wutausbrüche vorprogrammiert. 
„Deutschland sucht den Superstar“ gehörte zu den emotionalen Höhepunkten der Show. Die geschickte Fragetechnik einer Moderatorin kann emotionale Situationen hervorbringen oder noch steigern. Durch den Einsatz stilistischer Mittel wie gefühlvoller oder spannungssteigernder Musik, durch Großaufnahmen weinender Angehöriger oder durch detaillierte Dokumentationen und häufige Wiederholungen dramatischer Szenen sollen auch bei den ZuschauerInnen Emotionen geweckt und ihr Mitgefühl mit den im Fernsehen gezeigten Menschen noch gesteigert werden.

Intimisierung: Docu Soaps und Reality Soaps brechen schonungslos in die Privatsphäre der Menschen ein. Erstere, indem sie Aspekte des privaten Alltags filmt und so der Öffentlichkeit preisgibt, letztere, indem sie die Menschen in ihren Charaktereigenschaften und Handlungen entblößt. In ihnen leben „normale“ Menschen vor, wie man besondere, häufig schwierige Situationen meistert oder mit zwischenmenschlichen Konflikten in der Beziehung und zwischen Freunden umgeht. Vor allem die Privatsender bevorzugen dabei Themen, bei denen Menschen möglichst viel nackte Haut zeigen oder Sexualität eine Rolle spielt ${ }^{29}$. Was früher noch eindeutig im privaten Lebensbereich lag, wie persönliche Probleme, Sexualität oder die Gestaltung zwischenmenschlicher Beziehungen, wird beim Realitätsfernsehen zum öffentlichen Thema (vgl. Bente/ Fromm 1997, 20). „Tabubruch als Programm?“, die im Titel eines Bandes gestellte Frage, der sich mit der Vermischung von Öffentlichem und einst Privatem beschäftigt, kann für das Reality TV und seine Genres bejaht werden (vgl. Herrmann/ Lünenborg 2001). Tabubrüche können dabei sowohl einen aufklärerischen Impuls geben, wie etwa die Grimme-Preis-gekrönte Docu Soap „Abnehmen in Essen“ (WDR) zeigt, oder aber bestehende Vorurteile und Diskriminierungen verfestigen, wie es Friederike Herrmann (2002) für die Darstellung Bisexueller in den Talk Shows analysiert.

Stereotypisierung: In Docu Soap und Reality Soap werden nur ausgewählte Charakterzüge und Handlungen der ProtagonistInnen gezeigt und insbesondere einzelne Eigenarten betont und herausgestellt. Durch das Missverhältnis von gefilmter Lebenszeit und gezeigter Fernsehzeit ist es unmöglich, die Persönlichkeiten umfassend sichtbar werden zu lassen. Die damit gegebene Tendenz, die DarstellerInnen eher oberflächlich und stereotyp erscheinen zu lassen, wird durch eine spezifische Komprimierung des Materials und eine aus dem Gesamtkontext gerissene Präsentation weiter verstärkt. Beispielsweise repräsentierte Darstellerin Manu aus der ersten Staffel von „Big Brother“ die „Zicke“, Hanka aus der zweiten Staffel die „Hexe“. Die psychologischen Ursachen für das Übergewicht der beiden Schwestern Sabrina und Susanne Begic in „Abnehmen in Essen“ werden zwar angesprochen, jedoch nicht tiefergehend erörtert. Eine differenzierte, vielschichtige Darstellung der Charaktere ist nicht das Ziel von Docu Soaps und Reality Soaps. Eine umfassende Bearbeitung von Problemen wie auch eine sensible unverwechselbare Charakterzeichnung führte dazu, dass der Medientext für die Zuschauenden weniger produzierbar und damit wenig populär wäre (vgl. Fiske 1994, 103ff.). Gerade die Überzeichnung der Charaktere und die oberflächliche Darstellung von Problemen ermöglicht es den verschiedenen Gruppen von Zuschauenden, diese Vorlagen mit eigenen Bedeutungen zu füllen.

In jedem Fall gehört die Stereotypisierung der Handlung und der Darstellenden zu den gemeinsamen formativen Kennzeichen des narrativen und performativen Reality

29 Beispielsweise die zwischen 2001 und 2002 ausgestrahlten Reality Soaps „House of Love“ (RTL), „Girlscamp“ (SAT.1), „Expedition Robinson“ (RTL II), „Versuchung im Paradies“ (RTL), „Reeperbahn“ (RTL II). 
TV. Die erzählten Geschichten werden fast immer in kurzer Zeit dargestellt und zwingen zur Reduktion komplexer Zusammenhänge. ${ }^{30}$ Eine differenzierte Charakterentwicklung und -darstellung wird dadurch erschwert. Häufig kommen Klischees, stereotype Darstellungsmuster und standardisierte Handlungsabläufe vor (dies gilt für Reality TV, aber auch für Soap Operas oder viele Romane, vgl. Wegener 1994, 77ff.).

Dramatisierung: In den Docu Soaps und den Reality Soaps sorgt die Dramaturgie für besonders spannungsgeladene Momente. Beispiele dafür liefert die Nominierungs-Zeremonie der KandidatInnen im „Big Brother-Haus“, wer als Nächstes den Container verlassen muss, aber auch die komplizierte und gefährliche Geburt von Zwillingen (beispielsweise „Schnulleralarm“, RTL II). Die dabei gewählten Stilmittel entstammen der fiktionalen Serie oder dem Spielfilm, doch der Stoff kommt aus der Wirklichkeit. Alle Genres des Reality TV bereiten Ereignisse, gleich welcher Natur, dramatisch auf. Dramatik wird beispielsweise beim gewaltzentrierten Reality TV durch den Einsatz der „Living Camera“ erzeugt, ebenso durch spannungssteigernde Musik, schnelle Schnitte, überraschende Szenenwechsel und eine möglichst zugespitzte Darstellung des Ereignisses. Im Gerichtssaal sorgt die direkte Konfrontation der Streithähne für dramatische Momente, in denen Beziehungen beendet oder Ergebnisse von Vaterschaftstests bekannt werden. Bei „Deutschland sucht den Superstar“ wird erst im letzten Moment verkündet, wer die Show verlassen muss, so dass für Kandidatinnen wie Zuschauer die Spannung ins Hochdramatische steigt. Dieser „Entscheidung“ ist eine eigene Sendung gewidmet, die in der letzten Folge der ersten Staffel am 8. März 2003 mehr als 10 Millionen Zuschauer anlockte, was um diese Zeit nach Mitternacht einem sagenhaften Marktanteil von fast 62 Prozent entsprach (vgl. Hoff 2003, 19).

\section{Fazit}

Reality TV ist ein höchst lebendiges Sammelbecken erfolgreicher Formate, das sich in den letzten zehn Jahren rasant entwickelt und stetig ausdifferenziert hat. Das älteste dazu gehörende Genre, gewaltzentriertes Reality TV, hat heute seine Vorrangstellung gegenüber zahlreichen neuartigen Formaten eingebüßt. Reality TV hat in den 90er Jahren ein Stück Fernsehgeschichte geschrieben und ist längst mehr als eine Modeerscheinung. Um seine Entwicklung zur Genrefamilie angemessen zu erfassen, haben wir - angeregt durch Keppler - einen aktuellen Definitionsvorschlag für das narrative und das performative Reality TV vorgelegt und eine weiter gehende Klassifizierung vorgeschlagen. Zwischen dem ersten Boom 1992 und dem Fernsehen des beginnenden 21. Jahrhunderts können demnach elf verschiedene Genres des Reality TV identifiziert werden, wovon vier Vertreter des narrativen und sieben des performativen Reality TV sind.

Mit dem Boom der in diesem Beitrag besonders fokussierten Docu Soap und Reality Soap im Übergang zum 21. Jahrhundert zeigte sich besonders, dass das Reality TV von der Medienentwicklung stark beeinflusst war und diese wiederum weiter vorantrieb. Dabei blieben die Docu Soaps stets im Schatten der weit mehr Aufsehen erregenden Reality Soaps. Beide Genres stehen exemplarisch für die Veränderungen und die formativen Merkmale des Reality TV. Danach zeichnet sich die Genrefamilie vor allem durch inhaltlich markierte Grenzübertretungen aus: die Verschränkung informierender

30 Kein reales Gerichtsverfahren kann auf die Standardlänge von 20-30 Minuten verkürzt werden. Doch die Analyse tiefer gehender Ursachen und Motive für eine Tat sind bei Gerichts-Shows gar nicht erwünscht. 
und unterhaltender, inszenierender und authentischer, alltäglicher und außergewöhnlicher Bestandteile. Zugleich weisen die Genres des Reality TV eine Anzahl gemeinsamer Inszenierungsstrategien auf. Zu diesen Stilmitteln gehören Personalisierung, Emotionalisierung, Intimisierung, Stereotypisierung sowie Dramatisierung. Weil sich das Reality TV momentan besonders rasant entwickelt, ist diese Zusammenstellung der die Genrefamilie kennzeichnenden Merkmale sicher nicht im Sinne eines statischen, abgeschlossenen oder eindeutigen Kanons zu verstehen. Vielmehr versteht sie sich als Zwischenbericht, als Bilanz, von der ausgehend neue Forschungsperspektiven entwickelt werden können.

\section{Literatur}

Balke, Friedrich (Hg.) (2000): Big Brother - Beobachtungen. Bielefeld: Transcript.

Baudrillard, Jean (1978): Agonie des Realen. Berlin: Merve-Verlag.

Bente, Gary/ Fromm, Bettina (1997): Affektfernsehen. Motive, Angebotsweisen und Wirkungen. Opladen: Leske + Budrich.

Bleicher, Joan Kristin (1999): Fernsehen als Mythos: Poetik eines narrativen Erkenntnissystems. Opladen/ Wiesbaden: Westdeutscher Verlag.

Bleicher, Joan Kristin (2002): Formatisiertes Privatleben: Muster der Inszenierung von Privatem in der Programmgeschichte des deutschen Fernsehens. In: Weiß, Ralph/ Groebel, Jo (Hg.): Privatheit im öffentlichen Raum. Medienhandeln zwischen Individualisierung und Entgrenzung. Opladen: Leske + Budrich: S. $207-246$.

Böhme-Dürr, Karin/ Sudhold, Thomas (Hg.) (2001): Hundert Tage Aufmerksamkeit: Das Zusammenspiel von Medien, Menschen und Märkten bei „Big Brother“. Konstanz: UVK Verlagsgesellschaft.

Eggert, Jeannette (1999): Die Docusoap. Unveröffentlichte Diplomarbeit der Hochschule für Film und Fernsehen Konrad Wolff, Berlin-Potsdam.

Feige, Marcel (2001): Big Brother-TV: Wie Reality Soaps das Fernsehen verändern; mit dem ultimativen Reality-TV-Lexikon: Echte-Leute-TV von A bis Z. Berlin: Schwarzkopf \& Schwarzkopf.

Fiske, John (1994): Understanding Popular Culture. London/ New York: 2. Auflage, Routledge.

Fiske, John (1997): Television Culture: popular pleasures and politics. London/ New York: Routledge.

Flicker, Eva (Hg.) (2001): Wissenschaft fährt „Taxi Orange“: Befunde zur österreichischen Reality-TV-Show. Wien: Promedia.

Fromm, Bettina (1999): Privatgespräche vor Millionen. Fernsehauftritte aus psychologischer und soziologischer Perspektive. Konstanz: UVK Medien.

Früh, Werner/ Kuhlmann, Christoph/ Wirth, Werner (1996) [Früh et al.]: Unterhaltsame Information oder informative Unterhaltung? Zur Rezeption von Reality TV. In: Publizistik Nr. 4, S. $428-451$.

Gehrau, Volker (2001): Fernsehgenres und Fernsehgattungen. Ansätze und Daten zur Rezeption, Klassifikation und Bezeichnung von Fernsehprogrammen. München: Fischer.

Graff, Bernd (2003): Deutschland sucht sehr sonderbar. In: Süddeutsche Zeitung vom 10. Februar, S. 10.

Herrmann, Friederike (2002): Privatheit, Medien und Geschlecht. Bisexualität in Daily Talks. Opladen: Leske + Budrich.

Herrmann, Friederike/ Lünenborg, Margret (Hg.) (2001): Tabubruch als Programm. Privates und Intimes in den Medien. Opladen: Leske + Budrich.

Hoff, Hans (2000): Sein als Schein. In: Süddeutsche Zeitung vom 27. März, S. 22.

Hoff, Hans (2003): Immer dran glauben! In: Süddeutsche Zeitung vom 10. März, S. 19.

Hohlfeld, Ralf (2000): Weniger Wirklichkeit war nie - Big Brother und die Tradition des RealityFernsehens. In: Weber, Frank (Redaktion): Big Brother: Inszenierte Banalität zur Prime Time. Münster: Lit-Verlag, S. 195 - 204. 
Keppler, Angela (1994): Wirklicher als die Wirklichkeit? Das neue Realitätsprinzip der Fernsehunterhaltung. Frankfurt am Main: Fischer.

Klaus, Elisabeth (1996): Der Gegensatz von Information ist Desinformation, der Gegensatz von Unterhaltung ist Langeweile. In: Rundfunk und Fernsehen Nr. 3, S. $402-417$.

Krüger, Udo Michael (1992): Programmprofile im dualen Fernsehsystem 1985-1990: eine Studie der ARD/ ZDF-Medienkommission. Baden-Baden: Nomos-Verlagsgesellschaft.

Krüger, Udo Michael/ Zapf-Schramm, Thomas (2002): Öffentlich-rechtliches und privates Fernsehen: Typische Unterschiede bleiben bestehen. In: Media Perspektiven Nr. 4, S. 178 189.

Lücke, Stephanie (2002): Real Life Soaps. Ein neues Genre des Reality TV. Münster u. a.: Lit.

Meyers Lexikonredaktion (Hg.) (1995): Meyers großes Taschenlexikon: in 24 Bänden. Band 2. Mannheim u.a.: BI-Taschenbuchverlag.

Mikos, Lothar/ Feise, Patricia/ Herzog, Katja/ Prommer, Elizabeth/ Veihl, Verena (2000) [Mikos et al.]: Im Auge der Kamera. Das Fernsehereignis Big Brother. Berlin: Vistas-Verlag.

Müller, Eggo (1995): Television goes Reality. Familienserien, Individualisierung und ,Fernsehen des Verhaltens'. In: montage/ AV Nr. 1, 85-106.

Neale, Steve (2001): Studying Genre. In: Creeber, Glen (Hg.): The Television Genre Book. London: bfi Publishing, S. $3-4$.

Neuberger, Christoph (1994): Was ist neu am neuen Fernsehen? In: Medium Nr. 2, S. 67 - 71.

Niggemeier, Stefan (2003): Unsere Leichen leben noch. In: Frankfurter Allgemeine Sonntagszeitung vom 26. Januar, S. 25.

Nolda, Sigrid (1996): Verdeckte Vermittlung. Partnervermittlungen im Fernsehen. In: Medien Praktisch Nr. 4, S. $26-28$.

O.V. (1992a): Adolf-Grimme-Preis in Bronze, Spezial, Die Fußbroichs. In: Grimme, Zeitschrift für Programm, Forschung und Medienproduktion, special März 1992, S. 8.

O.V. (1992b): Escht escht. In: Spiegel Nr. 51, S. 215.

O.V. (1994): Ware Leben. In: Spiegel Nr. 15, S. $225 f$.

Pressemappe zu „Durchboxen“, ARTE/ WDR, ohne Datum.

Pressemappe der ersten Staffel von „Ein Heim für alle Felle“, WDR, 2000.

Pressemappe zu „OP. Schicksale im Klinikum“, ZDF Presse Special, 1998.

Pressemitteilung zu „Reeperbahn!“, RTL II, ohne Datum.

Schweer, Martin K.W. (Hg.) (2002): Das Private in der öffentlichen Kommunikation: „Big Brother" und die Folgen. Köln: Von Halem.

Strittmatter, Judka (1994): Niemand muß lila Haare haben oder Tattoos. Interview mit dem Produzenten Markus Peichl. In: Berliner Zeitung vom 28./ 29 Mai, S. 35.

Turner, Graeme (2001a): The Uses and Limitations of Genre. In: Creeber, Glen (Hg.): The Television Genre Book. London: bfi Publishing, S. 4-5.

Turner, Graeme (2001b): Genre, Hybridity and Mutation. In: Creeber, Glen (Hg.): The Television Genre Book. London: bfi Publishing, S. 6.

Weber, Frank (Redaktion) (2000): Big Brother: Inszenierte Banalität zur Prime Time. Münster: LitVerlag.

Wegener, Claudia (1994): Reality TV. Fernsehen zwischen Emotion und Information. Opladen: Leske + Budrich.

Weischenberg, Siegfried (2001): Nachrichten-Journalismus: Anleitungen und Qualitäts-Standards für die Medienpraxis. Opladen: Westdeutscher Verlag.

Winterhoff-Spurk, Peter/ Heidinger, Veronika/ Schwab, Frank (1994) [Winterhoff-Spurk et al.]: Reality TV. Formate und Inhalte eines neuen Programmgenres. Saarbrücken: Logos-Verlag. 